\title{
Complex Pattern Formation in Sessile Droplets of Protein-Salt Solutions with Low Protein Content. What Substance Fabricates These Patterns?
}

\author{
Tatiana A. Yakhno \\ Department of Radio-physical Methods in Medicine, Institute of Applied Physics RAS, 46 Ulyanov Street, 603950, Russia
}

\begin{abstract}
Complex and elegant patterns of rosette, scallop, Chinese arrow and dendrite shapes on microscopic length scales formed as a result of salt-induced protein self-assembly in sessile drying droplets of protein-salt solutions were described in the literature. The authors are confident in protein nature of these patterns because they used fluorochrom-labeled albumin, and carried out their observations under luminescent microscope at the appropriate wavelength. But this point of view seems to be disputable. In this paper, some data are presented to highlight the salt nature of the above-mentioned patterns.
\end{abstract}

Keywords Salt Crystals Polymorphism, Desiccated Sessile Drops, Protein Patterns Formation

\section{Introduction}

Sessile drying droplets of complex solutions are natural objects for studying different dynamical processes, which lead to self-assembly of their components[1-9]. These processes are very important for understanding the mechanisms of colloidal instability[8,10,11], medical diagnostics $[12,13,14]$, dye coatings quality $[15,16]$, and nano-particle formation[17,18]. Thus, this is a rapidly developing area of basic science, which has a wide number of useful applications. Therefore, correct understanding and interpretation of pattern formation in drying drops is very critical. During the last 10 years we studied the self-organizing processes in sessile drying drops by means of manifold approaches, and we were surprised by the interpretation of the experimental data published in[19]. Recently we observed the same patterns in protein-salt solutions at low protein content (not more than $0.5 \% \mathrm{w})$, using not only BSA (68 kDa), but also $\operatorname{IgG}(150 \mathrm{kDa})$ and carbonic anhydrase $(30 \mathrm{kDa})[20,21]$. We generally supposed that salt crystals, which demonstrated a broad polymorphysm, fabricated these patterns. However, the authors of[19] enforced us to make some additional experiments for verification of our point of view. In the current paper we present our past and recent data concerning this problem.

\section{Experimental}

* Corresponding author:

tanya-yakhno@rambler.ru (Tatiana A. Yakhno)

Published online at http://journal.sapub.org/pc

Copyright (C 2011 Scientific \& Academic Publishing. All Rights Reserved
Following the authors of[19], we used $0.2 \% \mathrm{w}$ bovine serum albumin solution (BSA, $68 \mathrm{kDa}$, Sigma, USA) in physiological salt solution $(0.15 \mathrm{M} \mathrm{NaCl}$, chemically pure, "Reactiv, Inc.", Russia). Also a pure physiological salt solution was used as a subject for investigation. The BSA solution was prepared without buffering, a day prior to experimentation, refrigerated overnight and allowed to come to room temperature before testing. The samples under study were placed, using a micropipette, onto clean glasses in the form of drops $5 \mathrm{ul}$ in volume (6-8 drops for each sample), and let for drying under room conditions.

It is not a trivial task to distinguish protein and salt structures under microscope without special analytical techniques. But, it is possible to try to do so resting upon different physical properties of protein and salt. So, the first idea implied the following: if we denaturate albumin and stick it to glass surface, then we can put the glass into water and dissolve salt. So we can display the salt crystals topology. Albumin denaturation was done by intensive warming of glasses with dried drops $10 \mathrm{~min}$ above a flame of a spirit-lamp. After warming, the glasses were allowed to come to room temperature, and were rinsed in distilled water.

The second idea was based on different interaction of albumin and salt with ethyl alcohol. Ethyl alcohol denaturates proteins, but does not dissolve $\mathrm{NaCl}$ crystals. Therefore, the glasses with dried drops of BSA salt solutions were put into $70 \%$ alcohol and kept there for $30 \mathrm{~min}$. Then, the glasses were dried at room temperature, and were investigated under microscope. After that, the glasses were rinsed in distilled water and investigated repeatedly. The dried droplets preparation of $\operatorname{IgG}$ and carbonic anhydrase salt solutions 
were described in[21] and[20], respectively. Morphological observations were carried out using MBS-10 and LUMAM-I3 microscopes, as well as video camera - computer setup and digital camera "Cannon".

\section{Results and Discussions}

Figure 1 shows "protein patterns" as they were described in the paper[19]. The fact is that $\mathrm{NaCl}$ itself can form crystals of different morphology in drops during water evaporation (Fig. 2). The complex salt patterns look like the "protein" patterns in[19]. Each of them consists of a large-scale single crystal in the center, and a lot of small crystals in the form of "frills", which surround it concentrically. The reason for these differences seems to be in different conditions for crystal growth: initial formation of a large crystal leads to depletion a surrounding solution with salt, thus continued water evaporation does not reserve time and substrate for another large crystal growth. In this situation small concentrical crystal pattern formation is the only feasible way.

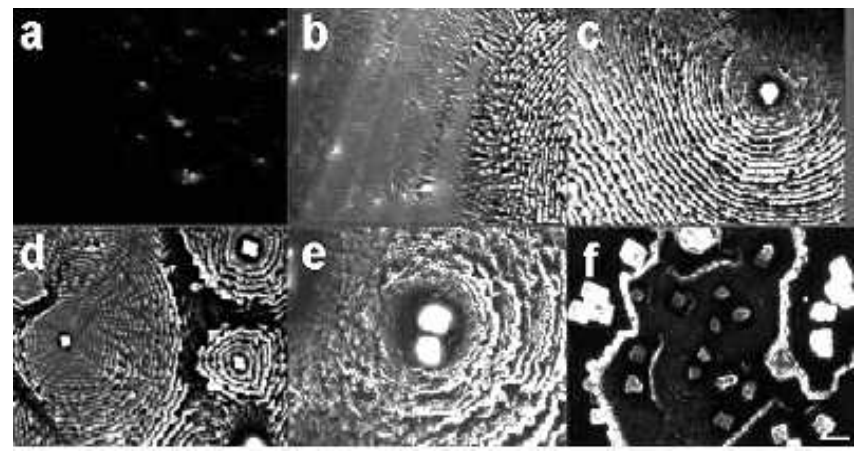

Figure 1. Fluorescence microscope images of protein patterns formed by slow evaporation under ambient conditions from $50 \mathrm{uL}$ of $1 \mathrm{mg} / \mathrm{mL}$ BSA Alexa Fluor 488 conjugate solution in PBS buffer with different salt concentrations: (a) 0 ; (b) $0.1 \mathrm{x}$; (c); $0.5 \mathrm{x}$; (d) $1 \mathrm{x}$; (e) $5 \mathrm{x}$; (f) $10 \mathrm{x}$. The scale bar represents 100 um[19, Fig. 3]

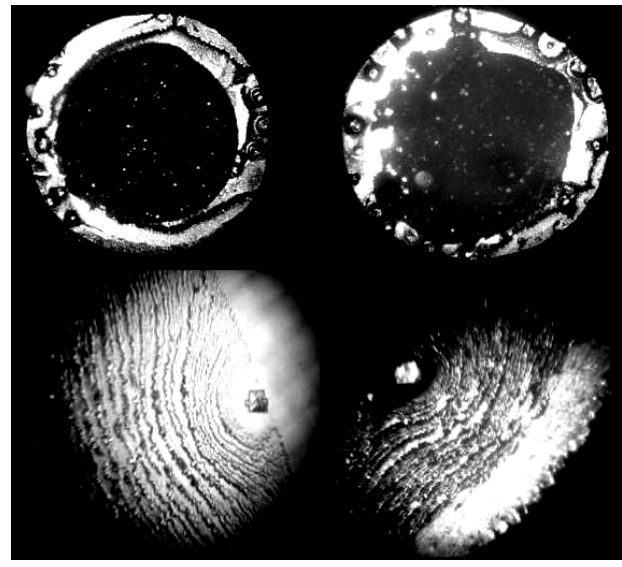

Figure 2. Optical microscope images of salt patterns in dried drops of $\mathrm{NaCl}$ water physiological solution. Upper line: magnification x 28 ; bottom line: magnification $\mathrm{x} 98$

The main distinction of the patterns in dried drops of salt solution before and after BSA adding (Fig.3) is their topology: due to the "coffee ring" phenomenon[1,2] in a pure physiological solution the patterns form mainly over a three-boundary line, whereas in BSA salt solutions these patterns lye in the drop centre. It is because protein adsorption to the glass surface takes place in protein salt solutions along with centrifugal stream[20]. Thus, when salt crystallization begins at the end of the drop drying process, protein bottom deposits can serve as seeds for crystal growth.

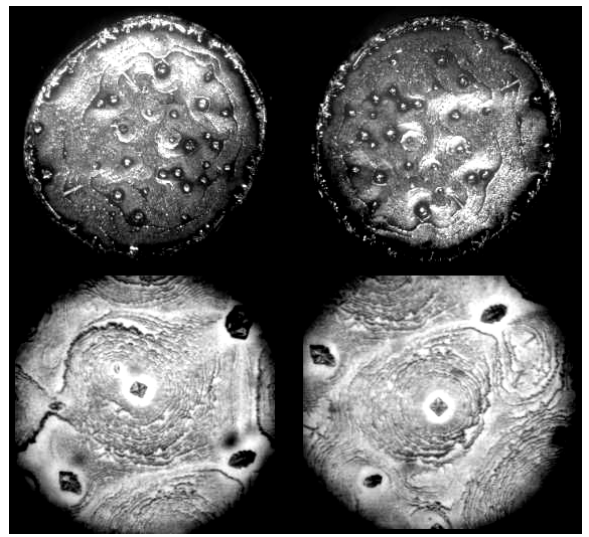

Figure 3. Optical microscope images of patterns in dried drops of $0.2 \% \mathrm{w}$ BSA solution in $\mathrm{NaCl}$ water physiological solution. Upper line: magnification x 28 ; bottom line: magnification $\mathrm{x} 98$

The glass warming procedure did not have significant influence on the pattern structure (Fig.4). Following water rinsing these structures vanished from the drop centre, and diminished essentially in height over the drop edge (Fig. 5).

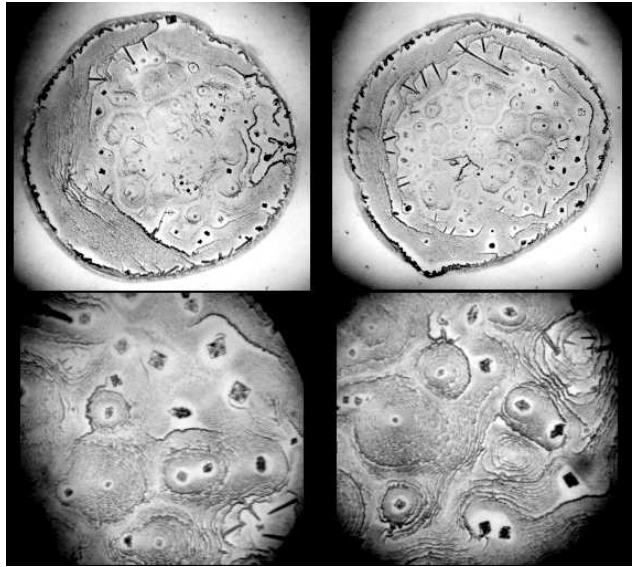

Figure 4. Optical microscope images of patterns in dried drops of $0.2 \% \mathrm{~W}$ BSA solution in $\mathrm{NaCl}$ water physiological solution after intensive warming of the glasses for $10 \mathrm{~min}$ above a flame of a spirit-lamp. Upper line: magnification x 28; bottom line: magnification x 98

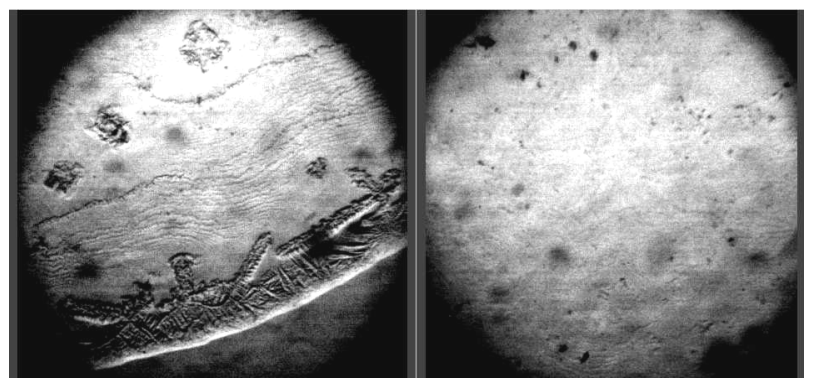

Figure 5. Optical microscope images of patterns in dried drops of $0.2 \% \mathrm{w}$ BSA solution in $\mathrm{NaCl}$ water physiological solution after intensive warming of the glasses for $10 \mathrm{~min}$ above a flame of a spirit-lamp and following water rinsing. Left - drop periphery, right - drop center (magnification x 56) 
Immersion of the glasses into ethyl alcohol led to albumin denaturation, so albumin formed microscopy-scale globules, which disappeared from the glasses partly. But, this procedure allowed observing true topology of salt patterns (Fig. $6)$.

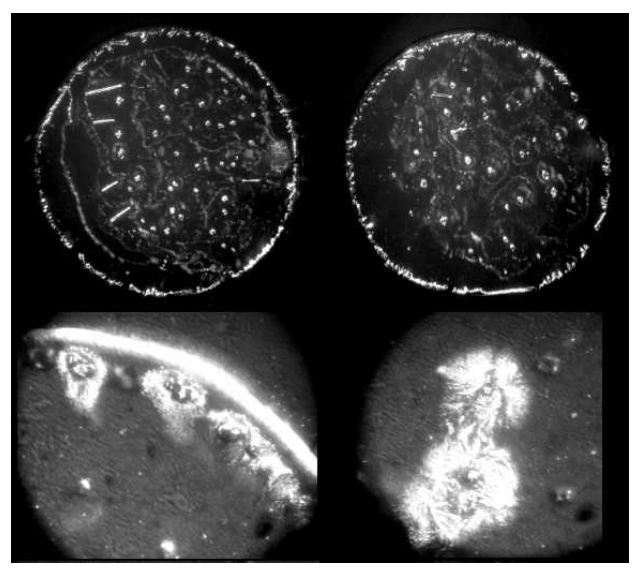

Figure 6. Optical microscope images of patterns in dried drops of $0.2 \% \mathrm{w}$ BSA solution in $\mathrm{NaCl}$ water physiological solution after keeping the glasses in ethyl alcohol for $30 \mathrm{~min}$. Upper line: magnification x 28; bottom line: magnification $\mathrm{x} 98$

These patterns, in turn, vanished fully after the water washing procedure (Fig. 7).

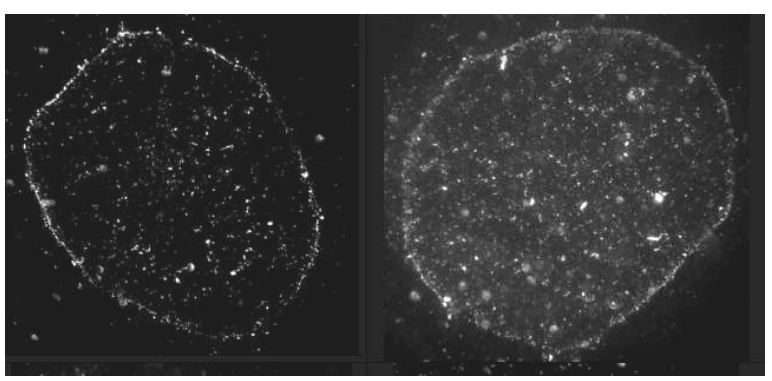

Figure 7. Optical microscope images of patterns in dried drops of $0.2 \% \mathrm{w}$ BSA solution in $\mathrm{NaCl}$ water physiological solution after keeping the glasses in ethyl alcohol for $30 \mathrm{~min}$ and following water rinsing (magnification x 28)

The same concentric patterns we observed also in IgG, and in carbonic anhydrase salt solutions (Fig. 8,9).

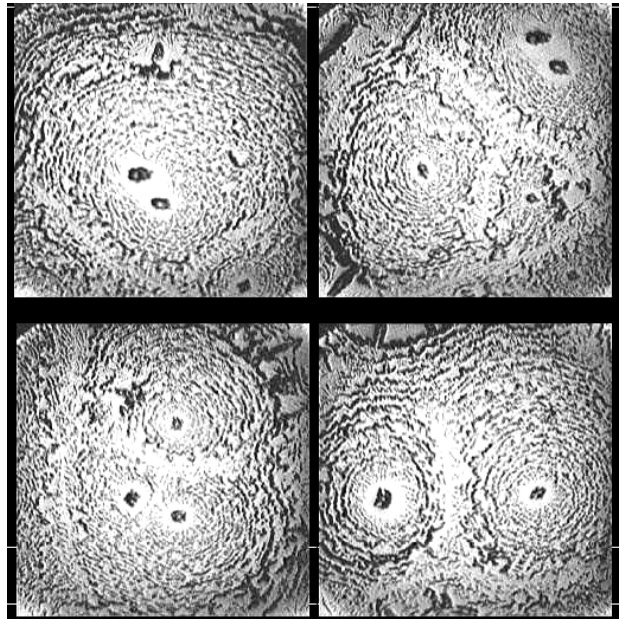

Figure 8. Optical microscope images of patterns in dried drops of carbonic anhydrase solution $(0.5 \mathrm{mg} / \mathrm{ml})$ in physiological salt solution (magnification $\mathrm{x}$ 140)

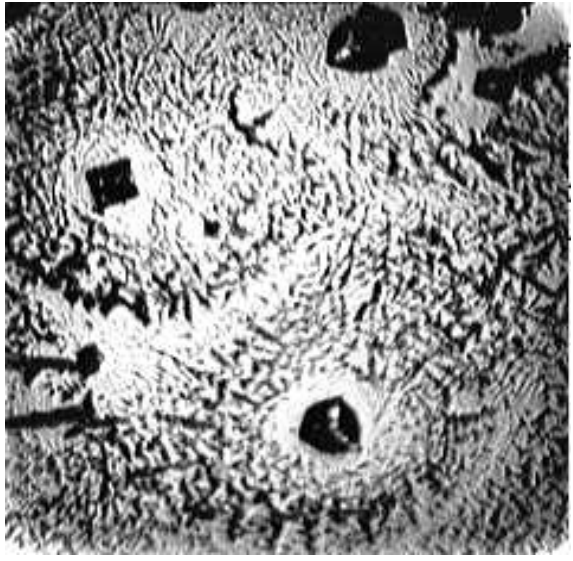

Figure 9. Optical microscope images of patterns in dried drops of $\operatorname{IgG}$ solution $(1.5 \mathrm{mg} / \mathrm{ml})$ in physiological salt solution (magnification $\mathrm{x} 140$ )

As to the protein mass content in these solutions, it is several fold less than the $\mathrm{NaCl}$ content, and up to $70 \%$ of protein mass moves to the drop periphery during drying[10], thus also confirming the assumption about the salt nature of these patterns.

And what about "specific fluorescence" of "protein patterns"? Undoubtedly, it is due to nonspecific light dispersion on the crystal surfaces. It is confirmed also by bright luminescence of large salt crystals in the centre of the "protein patterns"[Fig. 1]. The same "fluorescence", for example, can be achieved by lateral illumination of crystals by usual light, as is shown in Fig. 2 of this paper. Really, the same effect can be observed using visible light of any wavelength under appropriate light angulation. Thus, we suppose that all the discussions in[19] about the influence of rapid and slow evaporation, as well as protein and salt concentration, should be attributed to salt crystal growth. We are sorry about this delusion.

\section{Conclusions}

Complex pattern formation in diluted protein salt solutions was discussed. Using some simple methods, it was shown that these complex patterns were fabricated by salt. This conclusion contradicts the opinion of some authors. Further investigations by means of special modern analytical techniques should be useful.

\section{REFERENCES}

[1] R.D. Deegan, Phys. Rev. E. 61(2000) 475

[2] R. D. Deegan, O. Bakajin, T.F. Dupont, G. Huber, S.R. Nagel, T.A. Witten. Phys. Rev. E. 62 (2000) 756

[3] Y. Popov. Phys. Rev. E. 71 (2005) 036313

[4] H. Hu, R.G. Larson, Langmuir. 21 (2005) 3963

[5] H. Hu, R.G. Larson, Langmuir. 21 (2005) 3972 
[6] Yu.Yu. Tarasevich, D.M. Pravoslavnova. Eur. Phys. J. E. 22 (2007) 331

[7] W.D. Ristenpart, P.G. Kim, C. Domingues, J. Wan, H.A. Stone. Phys. Rev. Lett. 99 (2007) 234502

[8] V. Ragoonanan, A. Aksan. Biophys. J. 94 (2008) 2212

[9] R. Bhardwaj, X. Fang, D. Attinger. New J. Phys. 11 (2009) 075020

[10] T. Yakhno. J. Colloid Interface Sci. 318 (2008) 225

[11] T. Yakhno. Blood as a polydisperse system. Lambert Academic Publishing Gmb \& Co, Germany, 2011 (in Russian)

[12] V.N. Shabalin, S.N. Shatokhina. Morphology of human fluids. Khrizostom, Moscow, 2001 (In Russian)

[13] T. Yakhno, A. Sanin, V. Yakhno, A. Pelyushenko, N.A. Egorova, I.G. Terentiev, S.V. Smetanina, O.V. Korochkina, and E.V. Yashukova. IEEE EMB 24, 2 (2005) 96

[14] T. Yakhno. Natural Science. 3 (2010) 220. http://www.scirp.org/journal/NS/

[15] J. Park, J. Moon. Langmuir 22 (2006) 3506

[16] J. Wang, J.R.G. Evans. Phys. Rev. E. 73 (2006) 021501

[17] G. Liu, C. Zhang, J. Zhao, Y. Zhu. Langmuir. 24, 15 (2008) 7923

[18] M.L. Chabinyc, W.S. Wong. Method for aligning elongated nanostructures. US Patent 7838865. 11.23.2010

[19] G. Chen and G. Mohamed. Eur. Phys. J. E. 33, 1 (2010) 19

[20] T. Yakhno, V. Yakhno, A. Sanin, O. Sanina, and A. Pelyushenko. Tech. Phys. 49, 8 (2004) 1055

[21] T.A. Yakhno, V.G. Yakhno, A.G. Sanin, O.A. Sanina, A.S. Pelyushenko. Nonlinear Dynamics 39 (2005) 369 